\title{
Evaluating a new e-PDP tool and its relationship with personal tutoring
}

\section{Jane Rowe}

University of Exeter, UK

\section{Abstract}

The University of Exeter has used electronic tools to support the PDP process for students since well before the 2005 implementation of Progress Files. However, in 2007 high priority was given to the launch of a new e-PDP system to all staff and students through the long-established mechanism of the university's personal tutor system.

This paper explores, in the context of one academic school, the attempted integration between face-to-face 'developmental conversations' between tutors and tutees, and online recording of experiences and action plans by students. Whilst a fundamental change in the role of the personal tutor appears to have been accepted, the extent to which electronic tools are seen as an important part of the process is shown to be very much a live issue, centred on perceptions about ownership and responsibility.

The paper concludes that staff support for, and positive engagement among students with the principles of Personal Development Planning (PDP) do not necessarily translate into motivation to use online resources. Moreover, the findings of our project seem to confirm Richardson and Ward's (2005) observation that the terms 'e-Portfolio' or 'e-PDP', 'PDP' and 'Progress Files' are often used interchangeably and that implementation of online tools, particularly in personal tutor-led PDP programmes, must be managed carefully to avoid confusion between process and output.

\section{Key words:}




\section{Institutional context}

The University of Exeter is a research-intensive, 1994-group institution, spread across three campuses: two in the city of Exeter and one just over 100 miles away in Cornwall.

Exeter has almost doubled in size over the past ten years with 17,210 FTEs registered in 2009. At the time of writing, nine academic schools were in the process of re-structuring to become five large, cross-disciplinary colleges. The institution is highly focussed on becoming a 'Top 100' university on the global stage by 2015. Within the past five years there have been systematic reviews of schools' assessment and feedback strategies, employability and personal tutoring. It is therefore against a backdrop of rapid change that this project came into existence.

\section{PDP and the link with personal tutoring}

By 2005, the year that saw the mandatory introduction of Progress Files, transcripts were already being recorded electronically at Exeter. The university had also made significant steps towards articulation of PDP at an institutional level and most departments had responded in some way to the national requirement. Led by the university's Learning and Teaching Support Services (now Education Enhancement), a 'Framework for the Management of Skills' and a number of other PDP-related resources had been designed to aid students' reflection on their skills development (Dunne, 2001), and had been disseminated across schools. At the same time, however, a University-wide skills mapping project attempted to identify PDP-related activities already being undertaken within the curriculum and it was found that, whilst in some programmes transferable skill development opportunities and PDP activities were already well embedded, in others opportunities for students to reflect on their learning were more limited. In these cases, the PDP process was implicit rather than clearly communicated to students and to staff, and by 2002 the focus shifted towards the need for a university-wide mechanism to conduct student self-appraisal. It was at this point that, as a means of encouraging students to review learning across their whole programme, and in order to make the PDP experience more explicit, the personal tutor system became implicated as the primary vehicle to encourage students to reflect not only on their academic progress but also, on a more holistic level, on their broader and longer-term aims. 
Exeter had been one of the first universities to implement an electronic PDP system, and some schools were already beginning to trial it with students as a means of helping them prepare for meetings with their tutor. As Strivens (2006) has pointed out, the personal tutoring model of PDP delivery is appealing to a research-driven university; however, 'not all academics have the skills and aptitude for the tutoring role' and the tendency towards inconsistency can undermine the perceived value of processes which, as Fry et al. (2002) point out, already rest on concepts that are 'ill-defined', 'under researched' and dependent on a wide set of variables. Some tutors, whilst enjoying the personal contact with students, felt - and in some cases continue to feel - that the time involved in tutoring was and is not properly accounted for in workload models, and as a consequence ignored the broader personal development dimension of their role, restricting the level of their support to pastoral care and/or academic mentoring. Furthermore, some staff felt politically and ethically uncomfortable with what they perceived to be a change in the focus of the relationship with their tutees, signifying a move away from the traditional concept of academic guidance towards a relationship 'nested within the employability framework' (Clegg and Bradley, 2006). It is hardly surprising that at Exeter, in common with many other institutions, the notion of PDP as a process by which students' personal and academic skills might be 'applied' and somehow made relevant to the professionalised workplace (an external dimension of which tutors often have limited experience themselves) continues to be a contentious one in some departments.

\section{The 'recording' element of Progress Files: background to the Exeter experience}

The University of Exeter was an early adopter of technology to support the Progress File agenda. In as far back as 1999, a web-based profiling system, 'PESCA' (Prince, 2001), had been introduced in a number of academic departments. Not long afterwards a replacement system was launched, this time one that was specifically designed to link the student with their personal tutor. By 2005, PDP, as the system itself was known, was being used to some extent across all but one of the university's schools, but negative feedback about the system, and difficulties in supporting it centrally, made the institution wary of developing it further. In 2005 it was decided to design yet another system, this time one that was built on the technical infrastructure of the university's Student Record System 
(SRS). Given the long-established link between PDP and the nature of the support offered by personal tutors, it was felt important to provide an automatic link between a student's PDP and their personal tutor, and to build a system 'on top' of the University's SRS in order to facilitate that link. The expectation was also that, by using SRS, students would be building a resource that could link automatically to their transcript, and - potentially - to an ongoing record of their marks from assessments. The intention was to complement face-to-face tutor reviews by encouraging students to prepare their 'ePDP' in advance.

The development of these systems to sit alongside the provision of face-to-face personal tutor support, and the accepted practice of using the PDP acronym to 'name' these systems, had however led to a historic conflation of ideas about process and output. Whilst the majority of academic schools had, by the mid 2000s, made good progress in embedding aspects of personal development, skills and employability in the curriculum, the term PDP had largely become associated with the electronic system itself. In many cases this had become a hindrance rather than an enabling factor in terms of encouraging the embedding of the resource itself in programmes and modules.

The starting place for our NTFS project was therefore an interesting one. Whilst there was a need to evaluate usage of the new PDP tool (described in more detail in the following section), the project team also felt it necessary to deconstruct the complex assumptions around what students and staff interpreted as PDP and, in a sense, re-orient the institution's thinking around the PDP conundrum. Our project therefore sought to situate student and staff perceptions about the PDP (the process) in the broader framework of the personal tutor system. Our aim was to ascertain the perceived benefits but also the potential pitfalls and limitations of using an electronic resource to support the ongoing development of dialogue between tutors and tutees.

\section{Exeter's e-PDP tool}

Whilst still 'branded' with the PDP acronym, 'e-PDP' was radically different from the tool that had been used before. In response to student and staff feedback on the old system, it was decided to move away from asking students to reflect on a generic set of standard focussed learner questions about their progress. Staff had long complained that the old PDP system was not flexible or subject-specific enough. Moreover, students' responses to the formal question set of the previous system were often formulaic and lacked depth. It 
was hoped that e-PDP would give students much greater flexibility in articulating the experiences that were meaningful to them, whilst encouraging them to keep regular track of their learning beyond the minimum requirement for personal tutor meetings.

Through the more 'free form' design of the new system, it was hoped that students would conceptualise their learning experiences in more personal and creative ways, and be encouraged to see e-PDP as less of a once or twice a year form-filling exercise and more of a process that was designed to be ongoing - similar to a learning log or journal created and developed by themselves, albeit with the encouragement of their personal tutor. At the time the system was designed, there was already talk of the Higher Education Achievement Record (HEAR) being implemented, although the format of it was still unclear. By developing the new e-PDP system as an additional element of the university's Student Record System, it was envisaged that e-PDP might be used with the student's consent to provide evidence of active reflection and self-awareness, attributes that are well known to be important in graduate level employment, and that might therefore help to enrich a student's HEAR on presentation to a potential employer.

In addition to students' ability to conceptualise for themselves what they wished to record, the new e-PDP system allowed students full editing functions to review or add to their records at any time. In addition a skills-picking option would prompt them to cite the evidence for having gained a skill, just as they would have to in the context of an interview with a potential employer. Additional templates provided a structure, guidance and exemplars for SMART action plans, and also for personal statements, which some schools had expressed interest in asking their students to complete at the end of each academic year. Other features worthy of note were that students could flag the entries they wished to remain private, and also those they wished to include in a semi-formatted $\mathrm{CV}$, which could be provided as a print option and/or copied and pasted into a Word document for editing and polishing. 

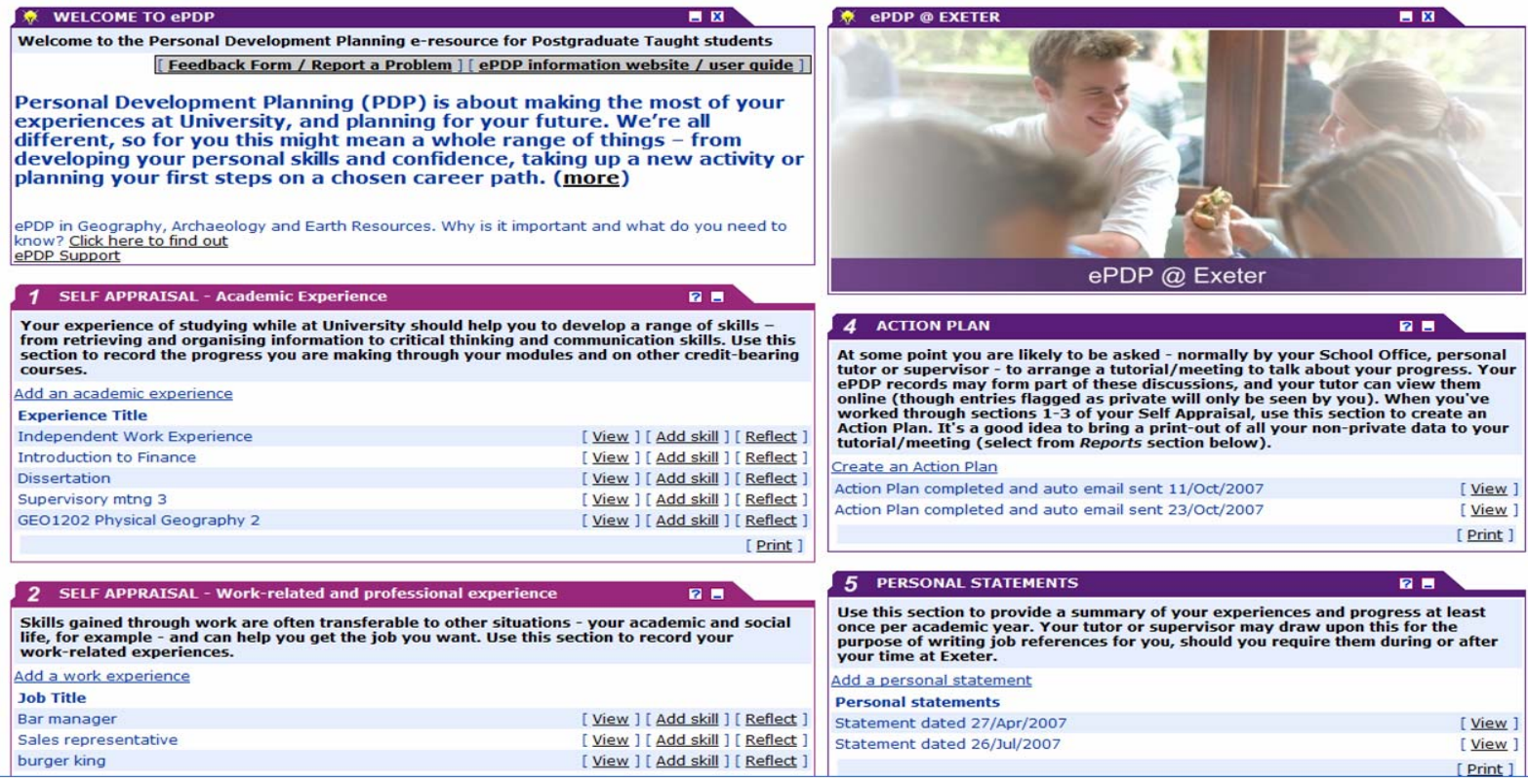

Figure 1. Example of a student's e-PDP home page.

The design phase completed, the system was finally launched in the academic year 200708 and all but one academic school agreed to use it with their students. A range of workshops were offered to personal tutors and also to learning support staff and administrators who it was anticipated were also likely to be giving additional support to students and staff during the transition from the old e-PDP system to the next. Sessions for students were also offered and some schools took advantage of these. In retrospect, it is clear that the implementation alone was an ambitious project. Given that at Exeter almost all academic staff have personal tutoring responsibilities, and that the personal tutor system was regarded as the principle vehicle for PDP across the university, the rollout was aimed at almost the entire academic population of the university - both students and staff. User guides were produced, and the university's PDP web pages were overhauled. All of this was complicated by the fact that for a time two systems continued to be supported centrally whilst the old e-PDP system was phased out.

Despite the new system's comparative sophistication compared to the previous one, it is easy to understand with the benefit of hindsight why the launch of yet another online Progress File tool was far from plain sailing! It soon became clear that for many students and academic staff, the result of strong 'systems-based' development was that the term PDP had come to signify the electronic repository itself rather than the process of reflection. To many academic staff it appeared that the institution was giving more 
importance to the record-creation element of the Progress File agenda than the human, face-to-face dimension which students, and often staff, tended to regard as far more meaningful. Others were still profoundly uncomfortable with the role of the personal tutor as anything more than a port of call for pastoral issues.

\section{The project}

This inter-connected bundle of tutor concerns around the Progress File agenda, combined with a timely request to evaluate a new Personal Development Tutorial Scheme (PDT) in the School of Engineering, Computing and Maths (SECaM), provided an opportunity to evaluate perceptions about the use of the new electronic PDP system. It also allowed investigation into possible linkages between the face-to-face practice of tutors talking with students about their development and the extent to which personal tutors felt comfortable with, what was for many of them, a rather different perception of the role than they had previously held.

In 2008-09, SECaM overhauled its personal tutor system. For many years, almost every member of SECaM's academic staff had held responsibility for anything from 10 to 30 personal tutees. However, one-to-one personal tutor meetings had been arranged on a relatively informal 'as needed' basis, and when they did take place (largely at the behest of the student rather than being proactively offered by staff) they were generally confined to academic content or pastoral matters. At the same time, a university-wide review of personal tutoring (2006-07) recommended further clarification of the developmental as well as pastoral role of personal tutors. The school decided to make changes accordingly, raising it to the top of its enhancement agenda for that year. Education Enhancement provided a series of staff development sessions; a school-specific Personal Tutor Manual was produced; a structured plan of PDT meetings was scheduled to take place throughout a student's degree programme, each with a clear purpose and agenda and initiated by the tutor; and tutorial meetings were formally recorded with the School Office. The student's responsibility was to prepare for these meetings using the e-PDP and to come to the meeting prepared to discuss it with their tutor.

The strategic drive behind the PDT Scheme was to clarify the scope and importance of PDP meetings as an important element of contact time, and to instigate some degree of 
consistency in what students could expect from their personal tutor. A year later, after SECaM merged with Physics and became the College of Engineering, Maths and Physics (CEMPS), it was decided that there should be a thorough evaluation of the PDT Scheme, whilst also comparing different tutoring practices with those of Physics. In response to this re-structure, the focus of the project shifted from not only an evaluation of the e-PDP tool, but also its relationship to the student and staff experience of PDP in the different subject areas.

\section{Methodology}

The link between e-PDP and the university's Student Record System (SRS) ensures that extracting quantitative data about students' use of the system is relatively straightforward. Our project was therefore able to draw on this to ascertain which functions of the e-PDP were used the most, how often students were on average using their e-PDPs and generally how successful different schools within the college had been in encouraging students to prepare for their tutor meetings using the e-PDP resource. System-generated data has its limitations, however, so it was decided to also run an online survey and focus groups to provide a richer picture of the students' experience of PDP. Finally, a series of semi-structured interviews was arranged with personal tutors themselves, again to explore their perceptions of PDP, of their subsidiary role as PDP advisers and the effectiveness or not, as they saw it - of their discipline's particular approach to PDP and personal tutoring in general.

\section{Project findings}

It was clear from the outset of this project that although the constituent schools within the college all had long established personal tutor systems and had established minimum requirements expected of tutors (including PDP guidance), the students themselves had very different approaches to PDP; expectations of their tutor; and the role and usefulness of the e-PDP system. Despite these differences, and according to the online survey data and focus group discussions, the stated aim of the e-PDP resource 'to help students prepare for their PDP tutorials' did not appear to have been achieved in any of the discipline groups. 
However, as is borne out by the emerging literature on e-portfolios from JISC and other sources, misconceptions abound concerning the perceived 'solution' of electronic resources to the implemention and embedding of PDP. Engaging students in the processes of PDP, let alone non-credit bearing work to prepare for tutorials, was never going to be something that a system on its own could miraculously achieve overnight, or for that matter, ever! Also, it did not help that Engineering and Maths had no previous culture of using e-PDP or e-Portfolio tools. In September 2007, when registrations on the old e-PDP system closed down after three years, only 10\% of Maths undergraduates had ever logged on to the system. In Engineering, there had been a higher uptake of 32\% but this was largely the result of a specific initiative led by a PDP champion within the department who has since left the university.

By comparison, when usage data from the new e-PDP system was extracted in a similar three-year period up to February 2010, there had been significantly higher uptake. Arguably, any increase in use of the new system might have been due to a developing awareness of PDP and related resources over the total six year period since provision of an online tool, rather than the tool necessarily being any better designed or its purpose more effectively communicated by tutors. It is clearly difficult to establish causality other than through an extensive longitudinal study.

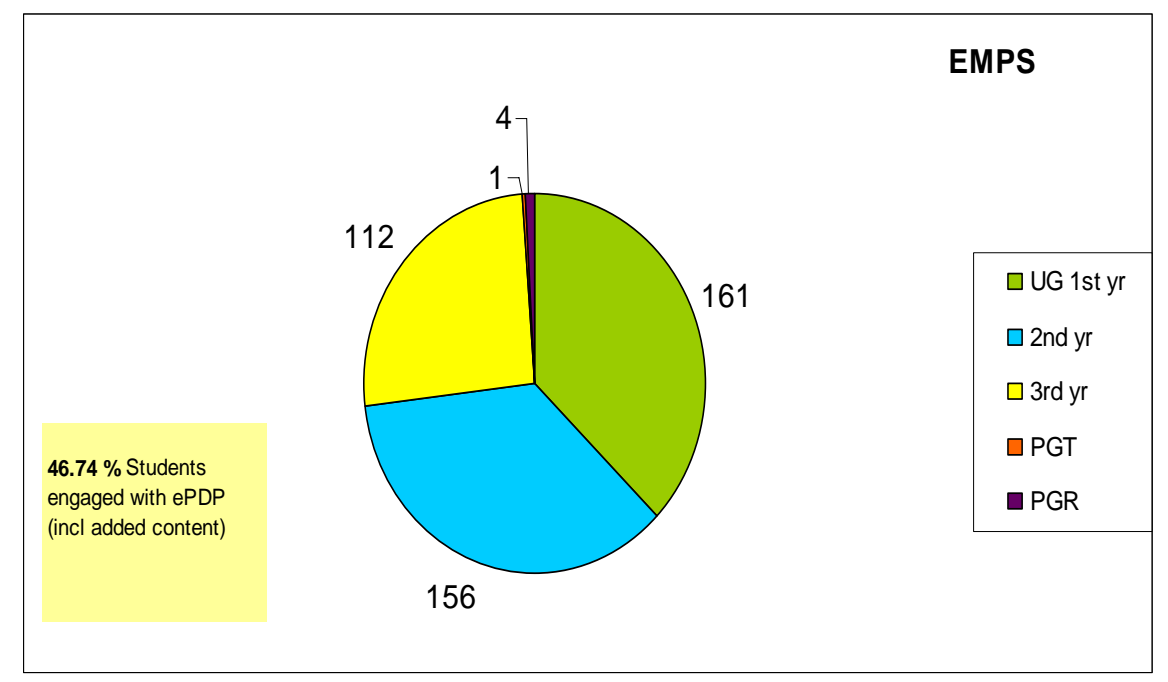

Figure 2. Engagement of Engineering and Maths students with e-PDP.

The results in Figure 2 show that in Feb 2010 a total of $46.7 \%$ of Engineering and Maths students had engaged with the e-PDP system, having not only logged on to the system but also added their own content. Usage was slightly higher in Years 1 and 2, which, given 
that the third years would have had much longer to discover the resource without any prompting from personal tutors or others, indicates that the school's PDT Scheme had indeed helped to raise awareness of the resource among students exposed to this higher level of tutoring. However, whilst this represents a significant improvement on the level of engagement with the old system, it was clear from the online survey data that the quantitative data alone needed to be interrogated in more detail through interviews and focus groups with students.

Despite the apparently positive statistics, the majority of Engineering and Maths students who responded to the project's online survey said they had never used the e-PDP. Those who had used the e-PDP said they had only used it 'when asked to do so'. When viewed as an activity that does not carry academic credit this is not surprising, but the transcripts from focus groups help to flesh out the ways in which students are instrumental about PDP in the context of a personal tutorial system. The Engineering students, for example, whilst recognising the long term value they would gain from keeping ongoing records of their experiences, were forthright about the strategic way in which they had to manage their workload and cited their experience of eight-hour days in the lab:

You're just not going to get people to write for half a page every week on what they've been doing...You've got to have your carrot or your stick. One of the two! (Engineering student)

These same students were positive, however, about the notion of including PDP as a credit-bearing element in their degree programme, pointing out that whilst PDP-related activities were embedded in some Engineering modules, they were somewhat constrained and did not necessarily enable them to reflect on their overall development at a deeper, more personal and holistic level:

There's Professional Studies and Skills Development 1 and 2. (Engineering student) 
Yes, and we already use the weekly log thing with our tutors. But that's just [in relation to] the particular project we're working on and unrelated to your entire university career, and obviously those things are then not thought of again. (Engineering student)

Whilst the students mentioned a range of skill development and CPD opportunities they were required to undertake as part of their academic programme (many of which were demanded by Engineering professional bodies as a requirement for professional recognition), they clearly perceived a lack of connection between these more embedded (and often assessed) PDP activities, such as compilation of a log book, and the more holistic discussions the school had hoped to engage them in through PDP tutorials. They spoke of the need to:

Bring it all together... Mix it all together. (Engineering student)

It's like, assuming you're doing this thing, you're keeping the log book and doing the CPD throughout the entire degree and it's accredited every year, then taking it to your [personal] tutor and the tutor reading it and advising based on the log book would work very easily. (Engineering student)

But whilst these students obviously recognised the value of PDP as a meaningful and useful activity in preparing them for employment, and wanted to see more attention paid to it at an earlier point in their academic career, they continued to see the most useful vehicle for reflection as the log book adopted by their module leaders, a familiar and assessed component of their degree programme:

Perhaps start it off in Year 1, not so much the e-PDP but keeping the log book. (Engineering student)

These students clearly advocated the use of tools that were associated with Atlay's (2009) integrated model of PDP already familiar to them through their academic experiences tools used that, for them, confirmed their academic and professional identity as engineers. The e-PDP vehicle for them was an irrelevance. That said, they strongly perceived the need for them to record their broader university experiences in some way, as well as the particular work required in their project-based modules, and acknowledged the need for 
this to be an assessed component of their programme, marked by someone who had the overview of their development:

At the moment log books are handed in to a single lecturer at the end of the year who marks them all. So maybe it should be an ongoing thing for the personal tutors. (Third year Engineering student)

Interviews with staff in both Engineering and Maths likewise suggested that whilst they did encourage students to engage with e-PDP, in doing so they were more than anything else 'observing protocols'. They often commented that students were using other means of recording their development, mainly through writing their own CVs and seeking guidance on those from the tutor. Far from the lack of engagement with e-PDP and lack of attention to the personal development of the students, it was very clear from these interviews that staff acknowledged the importance of the developmental relationship between tutors and tutees, but that the mechanisms through which this dialogue occurred, and the tangible outputs of the process, merely took a different form. As with the students, staff also often regarded the e-PDP as somewhat of an irrelevance. An Engineering tutor commented, for example, that reading a student's e-PDP either before or during a tutorial could even get in the way of more meaningful discussions with students:

You will often find that the written information is not the same, less full or less honest, than what they would tell you in person. (Engineering tutor)

The data provided by Physics students through the online survey presented a different but not entirely comparable view. In Physics, PDP tutorials were a recognisably different experience for students from their academic tutorials, and there was a long history of them being rigorously supported and monitored by senior management in the school. Personal tutors meet with their tutees on a weekly basis for academic tutorials in small groups, but have also for many years been encouraged to be proactive in setting dates for PDP tutorials and in following up incidences of non-attendance. PDP tutorials are intrinsically seen as part of the academic culture of the school and regarded as an important element of contact time. The PDP tutorials are also a mechanism to ensure student engagement with the employability agenda from early on in the student's programme. 
Such was the sense of ownership that Physics had developed their own PDP record system. In 2009 this school also adopted use of the new e-PDP and their students' engagement with it was cited by their Director of Education as being 'pretty much 100\%'. The online survey, however, combined with the results of the focus groups and staff interviews, again presented a more complex picture.

Whilst students from Physics appeared to be by far the biggest users of the e-PDP system, all of the groups surveyed rated it as the least important element of the PDP process.

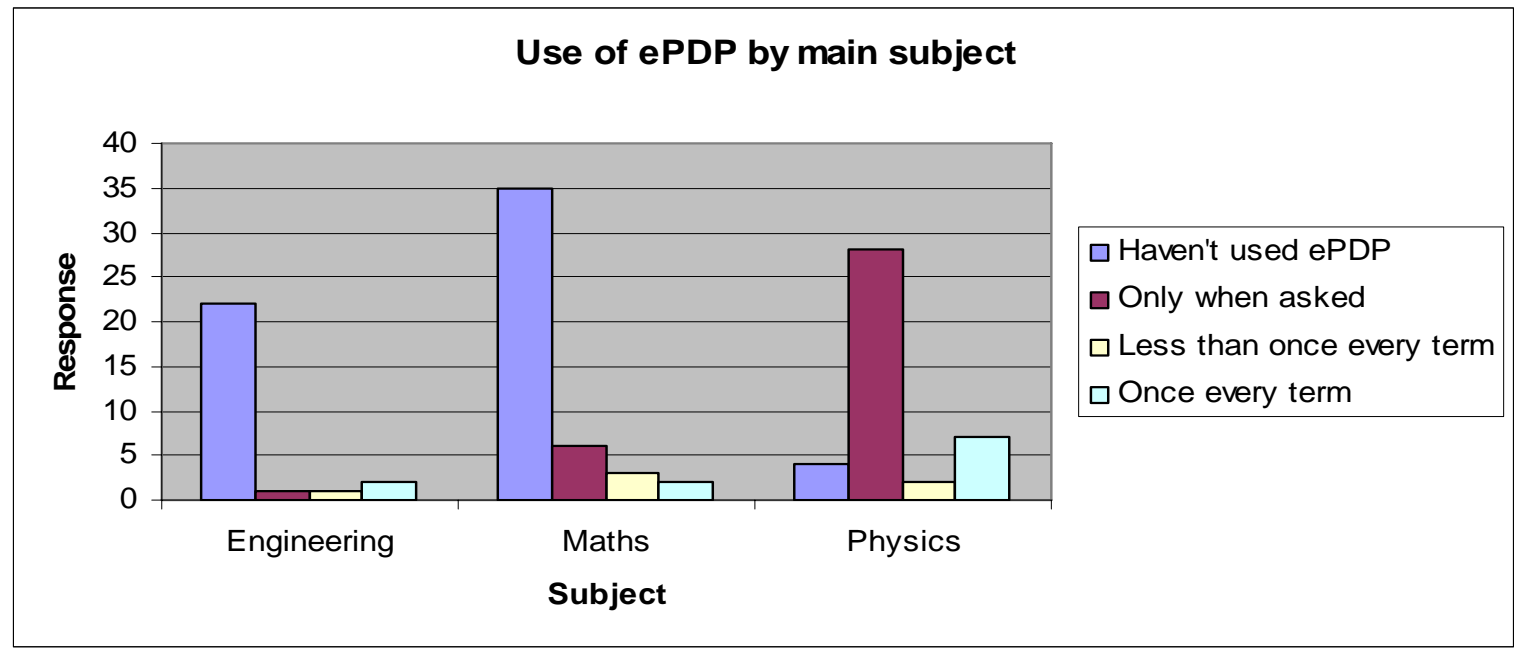

Figure 3. Use of e-PDP by main subject.

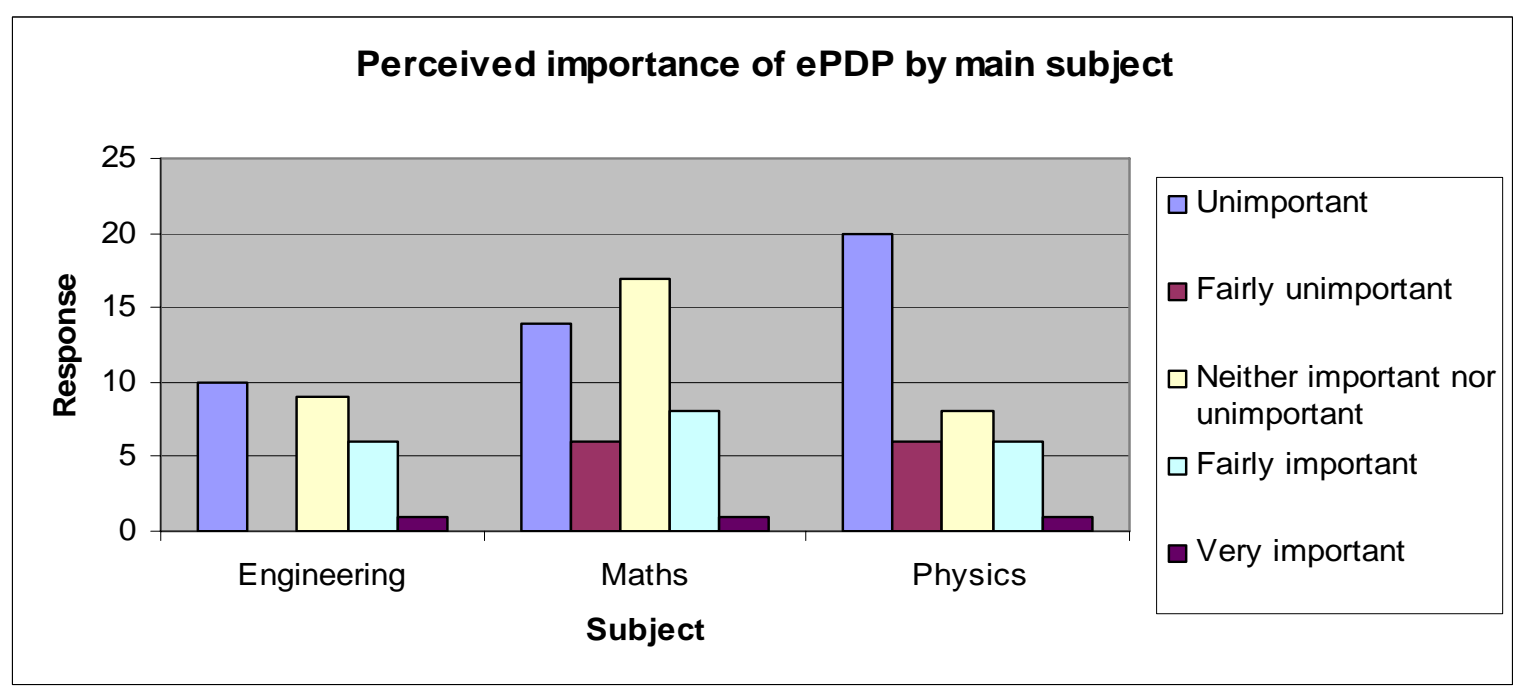

Figure 4. Perceived importance of e-PDP.

It appeared that despite the long history of using an e-PDP tool within the Physics department, the students did not appear to regard it as a useful learning aid. When asked 
a number of more general questions about the usefulness of the relationship with their personal tutor, however, the responses were consistently positive (Figure 5):

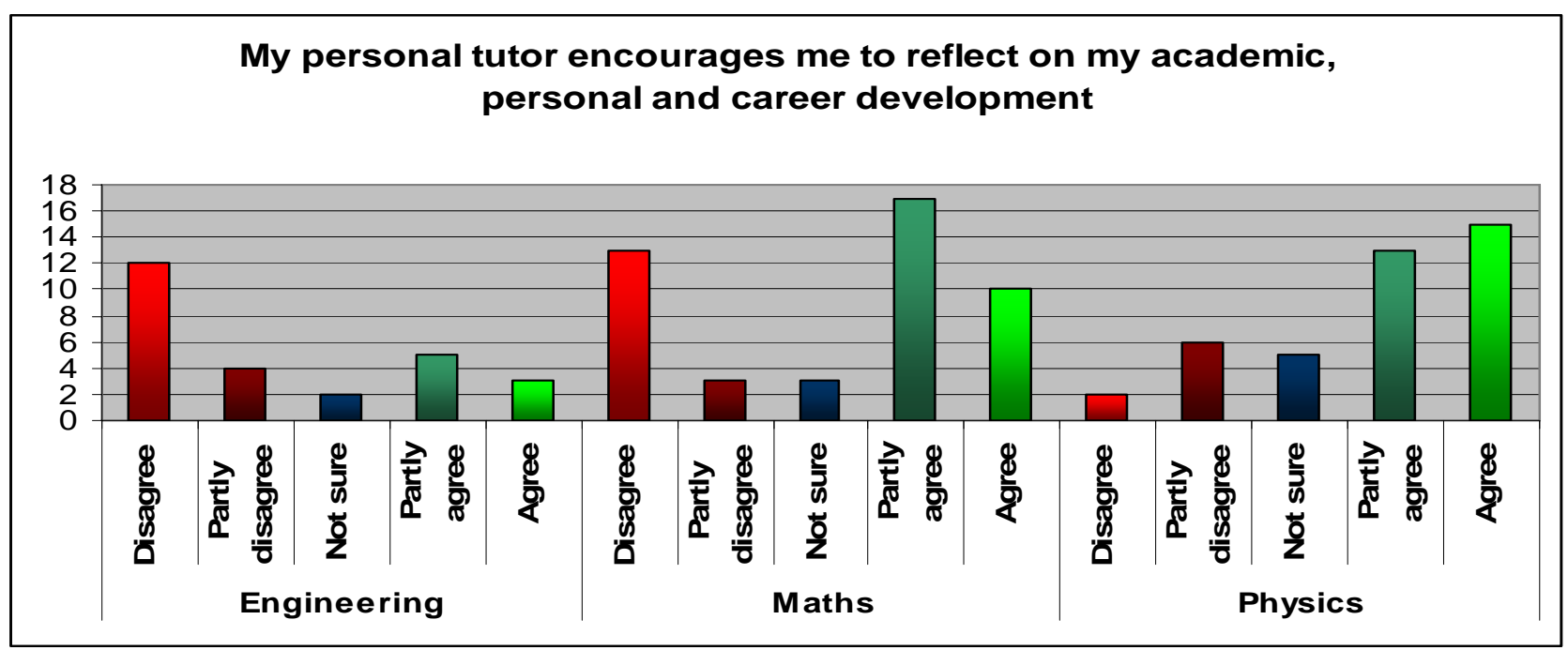

Figure 5. Usefulness of relationship with personal tutor.

When this was investigated in greater depth in the student focus group for Physics, a somewhat cynical view about the purpose of the system was revealed:

It's just a bit of bureaucratic nonsense really, isn't it? It's some way of someone being able to tick a box somewhere, so they can say 'Yeah, tutors made a connection with their tutees'. It's not a good way of trying to analyse how we're getting along on a personal level with our development. (Third year Physics student)

However, like the Engineering students, the Physics students were also keen to discuss ways in which face-to-face and online support for PDP could be improved. Inevitably, key to this was the way in which students were being motivated to engage with the process and indeed the resources associated with it:

Make it so that it's something that the student would do for their own sake, not because they have to. (Physics student)

Because there is an impression from the staff that it is this mechanistic format...that's immediately bestowed on all the students. (Physics student) 
They should make it seem more important to do it... There's a common perception that this is something that just has to be done and then brushed under the carpet. (Physics student)

Physics staff repeatedly commented on the 'lack of structure' provided by e-PDP. They argued that whilst they could see the rationale for enabling students to choose themselves the experiences they wished to record and reflect on, this 'free form' approach did not help students who found reflective practice difficult:

Physics students do not reflect, that's not what we do. I'm sure that's true of other Science students as well. You need to ask specific questions on that form, and then I guess a third to a half of students take it seriously...the others don't. Generally speaking, most students are fairly apathetic about [PDP], but the system itself doesn't help. I think it's very... it's way too touchy-feely! (Physics tutor)

Whilst the focus group for Physics in particular tended to dwell on the aspects of PDP and e-PDP that were not working effectively, it was clear from both these and the survey that, from the students' perspective, it was the nature of the personal relationship with individual tutors that was key to the value they ascribed to the process. The complementary model of academic tutoring in Physics, running alongside PDP tutorials, was certainly valued by students and there was a much clearer perception among students that their tutor has a developmental, as opposed to purely pastoral, role. However, it also seemed to be the case that where such roles were operating most successfully, it was more difficult to convince students of the merits of using the e-PDP resource - the process element of PDP aspect of PDP was clearly regarded as much more valuable and important than the output. The way in which the e-PDP was 'sold' to the students, i.e. as a strictly enforced, 'output driven' exercise with no credit attached to it, had therefore done little in terms of persuading them of the merits of using such a system. It might be construed that the richer the human relationships, the less meaningful will be the tools developed to support them. As Wenger (1998) neatly puts it, 'the tool can ossify activity around its inertness'. 


\section{Conclusion}

Whilst the debate continues as to whether the university's various e-PDP tools have been fit for purpose, our starting point has always been that simple usage statistics, based on the outputs are not in themselves adequate measures of engagement with the process that is PDP. Indeed, over-emphasis by institutions on the requirement for tangible recordkeeping element of the Progress Files, and on the importance of using technology to support the PDP process, can result in the notion that somehow PDP is deliverable through a simple electronic system of input/output, rather than through development of a rich and often highly negotiated relationship between tutor and tutee.

Among Maths, Engineering and Physical Science students at least, our research seems to bear out that whilst they valued the PDP support provided by tutors, they do not always see written reflection as a necessary or useful accompaniment to the process. Succinct, condensed formats such as CVs are recognised as more useful to their thinking process than more narrative, text-based accounts of the various experiences they've been exposed to.

Would it be the same in English or Psychology? Our data reveals that even where champions act as strong advocates for a centrally-provided system, and make a point of paying attention to students' PDPs before and during face-to-face tutorials, students will not necessarily perceive a benefit to using them. Indeed, among students and staff, the provision of a one size fits all, technology-based solution is often regarded as counterintuitive to the tutoring process, limiting rather than supporting open, developmental conversations between tutors and students, and almost inevitably failing to support the personal dimension of PDP.

In recent months, whilst the personal tutor system continues to be the insurance policy for ensuring engagement with PDP at all levels of the curriculum, there has been a significant move towards greater tailoring and embedding of PDP within modules - particularly in the first and final years. The need for further development of e-resources to support PDP, including the provision of fully-fledged e-Portfolios, continues to be a live issue but at the time of writing at least one school was developing its own specially tailored materials and systems in the hope that these will secure greater engagement from students and staff. It 
seems likely that over time others will follow, and in as much as ownership is essential for any successful adoption of PDP, such initiatives are undoubtedly being encouraged and supported. The extent to which locally-owned systems are seen by staff and students as relevant and useful, compared to previously used ones provided and maintained by the centre remains a question to be explored.

\section{Acknowledgement}

This paper is an outcome of the National Action Research Network on researching and evaluating Personal Development Planning and e-Portfolio practice project (2007-2010). The project was led by the University of Bolton in association with the University of Worcester and Centre for Recording Achievement, and in national collaboration with the University of Bedfordshire, Bournemouth University and University of Bradford. The project was funded by the Higher Education Academy, National Teaching Fellowship Project strand. More details about the project can be found at:

http://www.recordingachievement.org/research/narn-tree.html.

\section{References}

Atlay, M. (2009) 'Integrating PDP practice in the curriculum', in Yorke, M. (ed.) Personal development planning and employability. Revised edn. York: Higher Education Academy. Available at: http://www.heacademy.ac.uk/assets/York/documents/ourwork/employability/PDP a nd employability Jan 2009.pdf (Accessed: 27 August 2010).

Clegg, S. and Bradley, S. (2006) 'Models of personal development planning: practice and processes', British Educational Research Journal, 32 (1), pp. 57-76.

Dunne, E. (2001) 'Tracing the links, forging the patterns, or creating something from nothing: an account of policy, strategy, subject-led initiatives and research that have paved the way for PDP as a meaningful concept in the University of Exeter', Personal Development Planning: Institutional Case studies. Centre for Recording Achievement/Learning and Teaching Support Network Generic Centre. 
Fry, H., Davenport, E., Woodman, T. and Pee, B. (2002) 'Developing progress files: a case study', Teaching in Higher Education, 7(1), pp. 97-111.

Joint Information Systems Committee (2009), ePortfolios Infokit. (Accessed: 30 November 2010)

Prince, S.J. (2001) PESCA: introducing personal development planning at Exeter. Available at: http://www.ukcle.ac.uk/resources/pdp/exeter.html (Accessed: 7 July 2010).

Richardson, H.C. and Ward, R. (2005) Developing and implementing a methodology for reviewing e-Portfolio products. The Centre for Recording Achievement. Available at: http://www.jisc.ac.uk/uploaded documents/epfr.doc (Accessed: 5 September 2005).

Strivens, J. (2006) Transforming personal tutors into personal development tutors at the University of Liverpool. York: HEA. Available at:

http://www.heacademy.ac.uk/assets/York/documents/ourwork/tla/personal tutoring/ web0151 eCasebook transforming personal tutors into personal development $\mathrm{t}$ utors.pdf (Accessed: 27 August 2010).

Wenger, E. (1998) Communities of practice: learning, meaning and identity. Cambridge: Cambridge University Press.

\section{Author details}

Jane Rowe is a Learning and Teaching Adviser at the University of Exeter. 